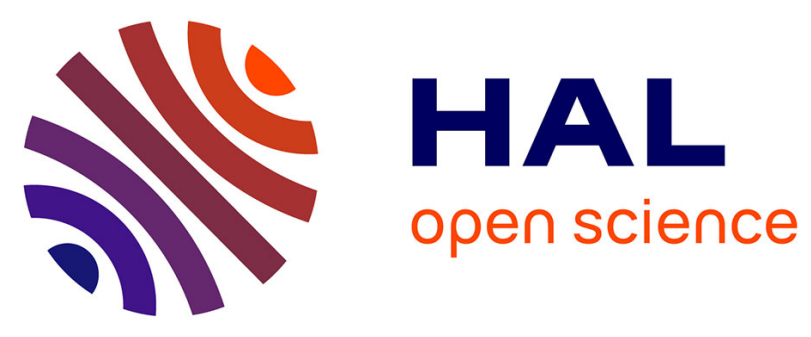

\title{
Region Tracking Algorithms on Laser Scanning Devices Applied to Cell Traffic Analysis
}

\author{
Aymeric Perchant, Tom Vercauteren, Fabien Oberrietter, Nicolas Savoire, \\ Nicholas Ayache
}

\section{- To cite this version:}

Aymeric Perchant, Tom Vercauteren, Fabien Oberrietter, Nicolas Savoire, Nicholas Ayache. Region Tracking Algorithms on Laser Scanning Devices Applied to Cell Traffic Analysis: Region Tracking Algorithms on Laser Scanning Devices Applied to Cell Traffic Analysis. IEEE International Symposium on Biomedical Imaging: From Nano to Macro (ISBI'07), Apr 2007, Arlington, United States. 10.1109/ISBI.2007.356838 . inria-00166610

\section{HAL Id: inria-00166610 https://hal.inria.fr/inria-00166610}

Submitted on 7 Aug 2007

HAL is a multi-disciplinary open access archive for the deposit and dissemination of scientific research documents, whether they are published or not. The documents may come from teaching and research institutions in France or abroad, or from public or private research centers.
L'archive ouverte pluridisciplinaire HAL, est destinée au dépôt et à la diffusion de documents scientifiques de niveau recherche, publiés ou non, émanant des établissements d'enseignement et de recherche français ou étrangers, des laboratoires publics ou privés. 


\title{
REGION TRACKING ALGORITHMS ON LASER SCANNING DEVICES APPLIED TO CELL TRAFFIC ANALYSIS
}

\author{
Aymeric Perchant ${ }^{1}$, Tom Vercauteren ${ }^{1,2}$, Fabien Oberrietter $^{1}$, Nicolas Savoire $^{1}$, Nicholas Ayache ${ }^{2}$ \\ ${ }^{1}$ Mauna Kea Technologies, 9 rue d'Enghien, Paris, France \\ 2 Asclepios Laboratory, INRIA, 2004 Route des Lucioles, 06902 Sophia-Antipolis, France
}

\begin{abstract}
In vivo and in situ confocal images are often distorted by motion artifacts and soft tissue deformations. To measure small amplitude phenomena on this type of images, we have to compensate for those artifacts. We present in this paper a Region Of Interest (ROI) tracking algorithm specialized for confocal imaging using a scanning device. Two different algorithms are presented: one based on the motion artifacts, and one based on affine registration. One typical application of this tool is developed: the blood velocity estimation inside a capillary on a moving organ. These first results show that the method permits accurate estimations of blood cell velocities even in presence of motion artifacts.
\end{abstract}

Index Terms - Tracking, in vivo confocal imaging, motion analysis, velocity measurement.

\section{INTRODUCTION}

Scanning imaging devices are becoming a standard tool to perform in vivo and in situ imaging both in research applications on living animals $[1,2,3]$ and in the clinical setting $[4,5]$. These new imaging technologies allow the acquisition and visualization of microscopic images at the cellular resolution in any part of the living body and in real time. The resulting video sequences can be used for visualization and quantification. The quest for noninvasiveness (organs should be neither touched nor removed) can lead to an instable motion of the tissue with respect to the imaging device. This can result in both motion artifacts and possible misquantifications. Both the miniaturization and the access difficulties are responsible for these possible motion artifacts.

A high frame rate can compensate for the resulting distortions in some cases. When the frame rate cannot be increased, motion artifacts appear on each frame of the video sequence. Because the acquisition device is a scanning device, these artifacts result in geometric distortions. Previous work has been done on the correction of these motion artifacts when creating larger images using video mosaicing techniques [6]. Following this work, we address in this paper the case when the user wants to focus on a small region in the living tissue that is difficult to stabilize. For instance in vivo and in situ acquisi- tion on the liver, the bladder or even the heart can be unstable. Such organs receive a growing interest among biologists to assess pharmaco-kinetics parameters of molecules, to screen the changing morphology of the anatomy, or to measure biodistribution parameters.

Due to the very specific type of images generated by in vivo confocal microscopy, classical image stabilization and registration techniques such as the one presented in $[7,8,9]$ could not be directly applied here. As a result, we developed a dedicated ROI tracking tool that takes into account the characteristics of in vivo confocal microscopy, and enables an automatic registration, analysis and quantification of an image sequence.

Cell traffic in micro vessels is an important research field in cellular biology and molecular imaging. Numerous algorithms have been developed to assess cell motion, or blood flow (e.g. $[10,11])$. To our knowledge there has been no attempt to perform blood flow velocity measurement on a sequence acquired in vivo with global tissue motion. This application illustrate the usefulness of the region of interest (ROI) tracking we propose.

In all the examples proposed hereafter, we used a Cellvizio developed by Mauna Kea Technologies (MKT) which is a complete fibered confocal microscope with a lateral and axial resolution comparable with a standard confocal microscope. It is based on the combination of 1) a flexible optical microprobe consisting in a bundle of tens of thousands of fiber optics, which overall dimensions are compatible with the accessory channel of a standard endoscope, 2) a proximal laser scanning unit, which combines the functions of light illumination, signal detection, robust and rapid scanning, and 3) a control and acquisition software providing real-time image processing.

Section 2 presents two algorithms to perform the tracking of a given ROI. The first one is based on the motion artifact compensation, and the second one on affine registration. Section 3 illustrates the application of the tracker on blood cell traffic measurement on a moving region. Section 4 concludes about the work presented herein. 


\section{REGION OF INTEREST TRACKER}

Two algorithms were developed that include either explicitly or implicitly motion distortion compensation.

\subsection{Scanning Artifacts and Motion Distorsions}

An interesting point of scanning imaging devices is that the output image is not a representation of a given instant, but a juxtaposition of points acquired at different times. Instead of motion blur, we get geometric distortions, e.g. a circle is distorted into an ellipse. During a rigid motion translation of a given object in the field of view, the transformation in image coordinates is a translation composed with a skew transformation [11]. This feature has been successfully used for red blood cell velocimetry on single images [11] and mosaicing of live video confocal images [6].

The scanning device we used has a fast horizontal scanning, and a slow vertical scanning. We showed in [11] that we could assume that the horizontal scanning has an infinite speed, and that the vertical scanning is described by the vertical position of the scanned point: $y_{s}(t)=v_{y} . t$, with $v_{y}=\frac{A_{y}}{t_{s}}$ where $A_{y}$ is the vertical amplitude, and $t_{s}$ the vertical scanning time, and $t$ the time.

\subsection{Motion Compensation Algorithm}

Let us suppose that the motion between two contiguous frames is a translation at speed $\tilde{\eta}=\left[\tilde{\eta}^{x}, \tilde{\eta}^{y}\right]$. A scanned line with vertical position $y$, will be sampled at the time $t(y)=t(0)+\frac{y}{v_{y}}$. During the scanning, a point $p=[x, y] \in I$ in the image reference plane will be sampled at position $p_{d}=\left[x_{d}, y_{d}\right]$ in the object reference plane. We note $\eta=\frac{\tilde{\eta}}{v_{y}}$ the normalized speed for the frame scanning. The position of $p_{d}$ is:

$$
\left\{\begin{array}{l}
x_{d}=x+(t(x)-t(0)) \tilde{\eta}^{x}=x+\left(y / v_{y}\right) \tilde{\eta}^{x}=x+\eta^{x} y, \\
y_{d}=y+(t(y)-t(0)) \tilde{\eta}^{y}=y+\left(y / v_{y}\right) \tilde{\eta}^{y}=\left(1+\eta^{y}\right) y .
\end{array}\right.
$$

For a given frame $k$, this linear transformation is noted $v_{k}$. Each point $p$ of a frame $I_{k}$ is mapped to a reference space coordinate system by the transformation $f_{k}: p \rightarrow p_{\text {ref }}$. Thus, we have:

$$
f_{k}(p)=r_{k} \circ v_{k}(p),
$$

with $r_{k}$ a rigid transformation. Between two frames $j$ and $k$, the transformation is given by:

$$
f_{j, k}(p)=v_{j}^{-1} \circ r_{j}^{-1} \circ r_{k} \circ v_{k}=v_{j}^{-1} \circ r_{j, k} \circ v_{k} .
$$

The estimation of the velocities is done using only the translation part of the $r_{j, k}$. This velocity is used in the following algorithm to compensate for the scanning distorsions. For each contiguous frames the following steps are performed:

1) estimation of the translation using a $2 \mathrm{D}$ normalized cross correlation,

2) estimation of the velocity from the translation,

3) computation of the distortion transformation,

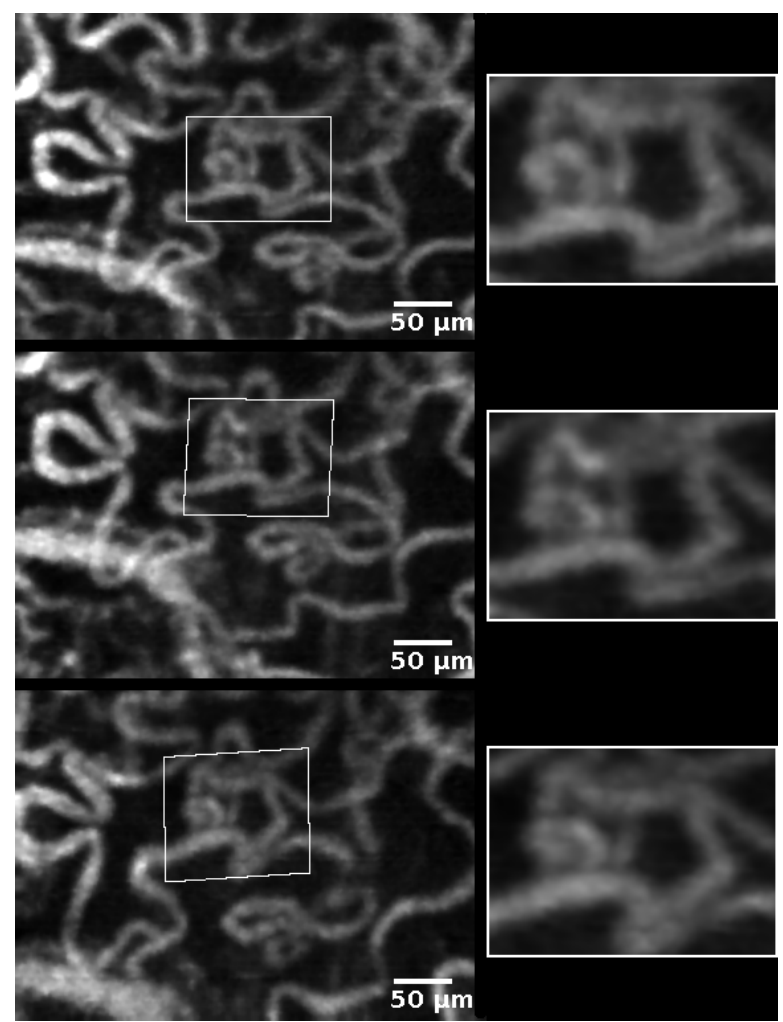

Fig. 1. Tracking a selected ROI on tumor vasculature acquired in vivo. The upper frame is the reference frame, the other rows represent the frames 11 and 15 at from a 200 frame sequence. The tracked ROIs are shown on the left column with the corresponding warped region on the right column.

4) optimization of the rigid transformation.

Fig. 1 shows an example of ROI tracking using the motion compensation algorithm. On the second row, a typical scanning distorsion is visible. On the last row, the transformation is a composition of a scanning distorsion, and a rigid transformation with a rotation.

\subsection{Affine Registration Algorithm}

Using Cellvizio device, the handheld probe can freely glide along a soft tissue, while keeping contact with it. Thus, the transformation may be composed with a possible rotation, or even a little scaling when the tissues are compressed. In this section, we choose to represent the transformation due to the laser scanning confocal device by an affine transformation.

To ensure a faster convergence in an optimization scheme, we initialized each transformation by the best translation found by a normalized cross correlation. For each frame, we choose to minimize the sum of squared differences: $\sum_{p} \| I(p)-$ $I_{k}\left(T_{k}^{a}(p)\right) \|^{2}$, with $T_{k}^{a}$ the affine transformation for frame $k$.

The optimization scheme was chosen to be fast and efficient. The ESM (Efficient Second order Minimization) [12] 
provides excellent results in real time robotic applications. We have adapted it to use an affine transformation instead of a projective one. The main advantage of the algorithm is to be a true second order optimization scheme with the complexity of a first order optimization scheme such as Gauss-Newton. Of course, other optimizers could be used.

Fig. 2 shows a result of the affine registration algorithm.

\section{APPLICATION TO CELL TRAFFIC ANALYSIS}

The ROI tracking was used to assess cell traffic in a capillary. The aim is to measure the blood velocity in a capillary. The user can select manually a rectangular ROI on the image. Fig. 2 shows the tracking of this region on a sequence acquired with a hand-held probe on a tumoral skin xenograft on a living mouse. Vessels were stained using dextran fluorescein from Invitrogen.

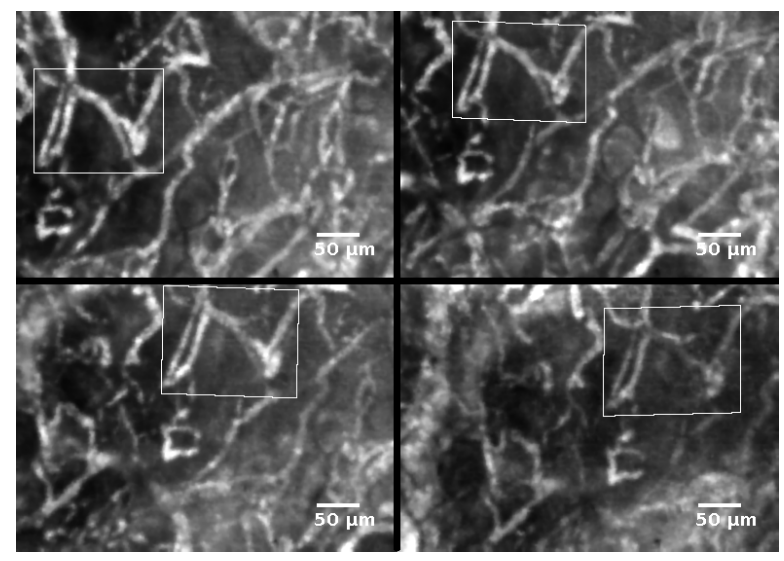

Fig. 2. ROI tracking using affine transformations: 4 frames from the same sequence are displayed with the registered ROI. Complete sequence includes 237 frames.

Using the tracking, we resampled each frame to compose a stabilized sequence. Three frames of this sequence of 5 seconds (acquired at $12 \mathrm{~Hz}$ ) are presented in Fig. 3.

On the temporal mean frame of the stabilized sequence, we have segmented the vessels using a $2 \mathrm{D}$ adaptation of the multi-scale tubular vessel detection algorithm of Krissian et al. [13]. We used this adaptation on the same type of images in [1]. The upper left frame of Fig. 3 shows the result of the detection: the medial axis and the vessel borders. The mean vessel diameter is $10.7 \mu \mathrm{m}$, which is roughly the size of a red blood cell.

The medial axis of the vessel in the ROI displayed in Fig. 3 was used to extract the vessel intensity in the center line. Two temporally contiguous lines are displayed in Fig. 4. The normalized cross correlation of these two lines are displayed in Fig. 5 together with the mean value of the correlation for all the pairs of contiguous frames. Fig. 6 shows the estimation of the velocity of the blood in the capillary.

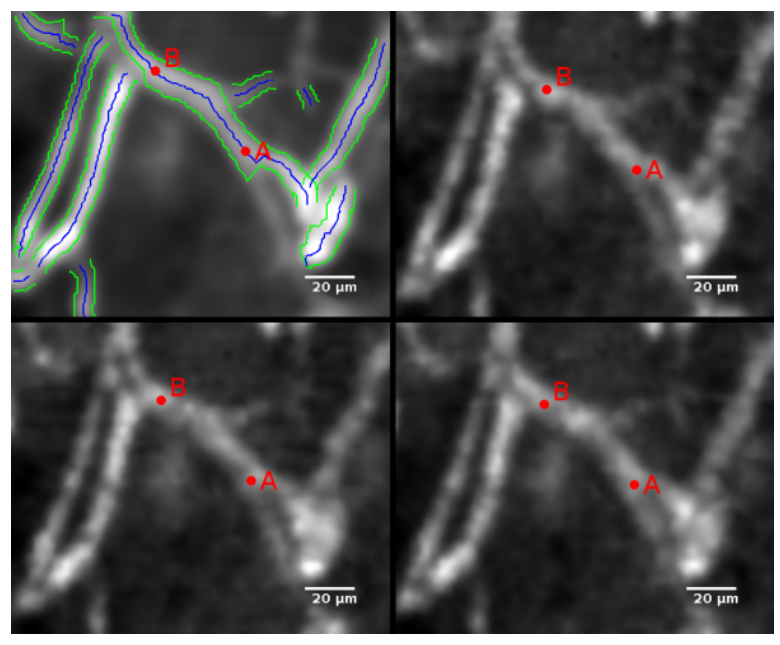

Fig. 3. Upper-left: vessel detection on the temporal mean frame after stabilization. Other images: three contiguous frames of the stabilized sequence $(12 \mathrm{~Hz})$. Blood velocity was acquired on the medial axis segment $[\mathrm{AB}]$.

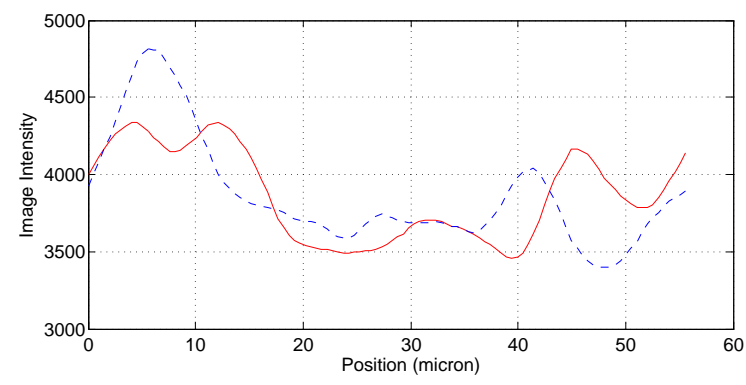

Fig. 4. The medial axis intensity of the same vessel on two contiguous frame. The correlation of the two signals is visible.

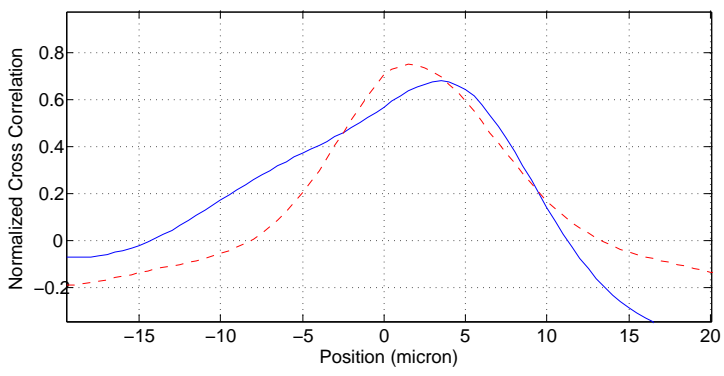

Fig. 5. The normalized cross correlation of the lines of Fig. 4 (plain line), with the mean value of the correlation for all the sequence (dashed line).

The range of velocities that we can address depends on the scanning period, and the amplitude. Typical values on the device we used are $12 \mathrm{~Hz}$ frame rate, with a field of view of 


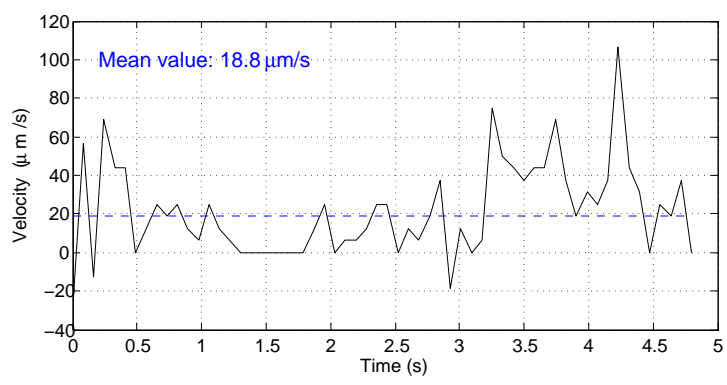

Fig. 6. Velocities computed using the correlation method on the registered ROI. The dashed line indicates the mean value of the velocities.

$0.5 \times 0.6 \mathrm{~mm}^{2}$, and a $3.5 \mu \mathrm{m}$ resolution. The velocity precision is given by the minimum translation observable between two frames: $\delta v=0.012 \mathrm{~mm} / \mathrm{s}$. The velocity interval computed using a maximum detectable translation of half the horizontal field of view is $[0,3.6] \mathrm{mm} / \mathrm{s}$. The same device can perform fast scanning at a frequency up to $200 \mathrm{~Hz}$ with the same resolution by reducing the vertical field of view. The velocity interval in this case is maximal when the vessel is horizontal: $[0,60] \mathrm{mm} / \mathrm{s}$ with a precision of $0.2 \mathrm{~mm} / \mathrm{s}$.

\section{CONCLUSION}

We presented a framework to accurately measure blood velocity inside a small capillary on a moving region of interest in a field of view. The region of interest is stabilized and resampled using a specialized tracking algorithm. Two versions of this algorithms were proposed based either on a specific scanning distortion estimation, or a global affine transformation. The motion compensation algorithm has stronger constraints, but is faster than the affine registration. The best algorithm will probably be application dependant. The two algorithms are currently beeing compared for performance evaluation. On the presented cell traffic application, a region was stabilized, and the capillaries were detected using tubular model-based segmentation. The medial axis signal level was extracted from the image. The spatio-temporal correlation was finally used to estimate the blood velocity in the capillary. Now that the feasibility of the framework has been proved, the next step is the validation on both numerical and real data.

\section{ACKNOWLEDGMENTS}

We would like to thank Ezio Malis from INRIA for very interesting talks about the ESM algorithm and its various applications. Images in Fig. 1 are courtesy of Anne-Carole Duconseille and Olivier Clément, Descartes Image, Small Animal Imaging Facility, Université Paris V, Paris, France. Images in Fig. 2 and 3 are courtesy of Raphaël Boisgard, Frédéric Ducongé and Bertrand Tavitian, CEA SHFJ, Inserm, U 803 Orsay France.

\section{REFERENCES}

[1] K. Y. Lin, M. A. Maricevich, A. Perchant, S. Loiseau, R. Weissleder, and U. Mahmood, "Novel imaging method and morphometric analyses of microvasculature in live mice using a fiber-optic confocal laser microprobe," in RSNA. Chicago, Il, USA: RSNA, 2006.

[2] P. Vincent, U. Maskos, I. Charvet, L. Bourgeais, L. Stoppini, N. Leresche, J.-P. Changeux, R. Lambert, P. Meda, and D. Paupardin-Tritsch, "Live imaging of neural structure and function by fibred fluorescence microscopy." EMBO reports, vol. 7, no. 1, pp. 1154-1161, sept 2006.

[3] N. Ayache, T. Vercauteren, G. Malandain, F. Oberrietter, N. Savoire, and A. Perchant, "Processing and mosaicing of fibered confocal images," in MICCAI Workshop on Microscopic Image Analysis with Applications in Biology (MI$\left.A A B^{\prime} 06\right)$, Oct. 2006, invited talk.

[4] L. Thiberville, S. Moreno-Swirc, T. Vercauteren, E. Peltier, C. Cavé, and G. B. Heckly, "In vivo imaging of the bronchial wall microstructure using fibered confocal fluorescence microscopy," American Journal of Respiratory and Critical Care Medecine, October 2006, doi:10.1164/rccm.200605-684OC.

[5] A. Meining, M. Bajbouj, and R. M. Schmid, "Confocal fluorescence microscopy for detection of gastric angiodysplasia," Endoscopy, 2007, in press.

[6] T. Vercauteren, A. Perchant, G. Malandain, X. Pennec, and N. Ayache, "Robust mosaicing with correction of motion distortions and tissue deformation for in vivo fibered microscopy," Medical Image Analysis, vol. 10, no. 5, 2006.

[7] G. Hager and P. Belhumeur, "Efficient region tracking with parametric models of geometry and illumination," IEEE Trans. Pattern Anal. Machine Intell., vol. 20, no. 10, pp. 1025-1039, 1998.

[8] M. Isard and A. Blake, "CONDENSATION-conditional density propagation for visual tracking," International Journal of Computer Vision, vol. 29, no. 1, pp. 5-28, 1998.

[9] C. Wilson and J. Theriot, "A correlation-based approach to calculate rotation and translation of moving cells," IEEE Trans. Image Processing, vol. 15, no. 7, pp. 1939-51, 2006.

[10] Y. Sato, J. Chen, R. Zoroofi, N. Harada, S. Tamura, and T. Shiga, "Automatic extraction and measurement of leukocyte motion in microvessels using spatiotemporal image analysis," IEEE Trans. on Biomedical Engineering, vol. 44, no. 4, pp. 225-236, april 1997. [Online]. Available: citeseer.nj.nec.com/35344.html

[11] N. Savoire, G. Le Goualher, A. Perchant, F. Lacombe, G. Malandain, and N. Ayache, "Measuring blood cells velocity in microvessels from a single image: Application to in vivo and in situ confocal microscopy," in Proc. ISBI'04, Apr. 2004.

[12] S. Benhimane and E. Malis, "Homography-based 2d visual tracking and servoing," Joint Issue of IJCV and IJRR on Vision and Robotics, 2007, to appear.

[13] K. Krissian, G. Malandain, N. Ayache, R. Vaillant, and Y. Trousset, "Model-based detection of tubular structures in 3D images," Computer Vision and Image Understanding, vol. 80, no. 2, pp. 130-171, 2000. 